Forum Psychoanal $2006 \cdot 22: 240-248$ DOl 10.1007/s00451-006-0287-4

○) Springer Medizin Verlag GmbH 2006
Siegfried Zepf · Saarbrücken

\section{Das Konzept der „psychischen Realität“}

or zwanzig Jahren urteilte Michels (1985, S. 515), dass fast siebzig Jahre nach Freuds (1916-17a, S. 383) Feststellung, dass „in der Welt der Neurosen die psychische Realität die maßgebende ist", dieses Konzept „eine zentrale Stelle in der psychoanalytischen Theorie und Behandlungstechnik" in dem Sinn beibehalten habe, dass es unverändert „das Gebiet der Psychoanalyse" definiere. Allerdings habe sich das, was heute als „psychische Realität" bezeichnet wird, verändert. Während Freud darunter eine biologische Realität, die Realität der „biologisch verwurzelten Triebe" verstanden habe (Michels 1985, S. 516), würde dieser Begriff heute relativ einheitlich im Hinblick auf „die innere Welt subjektiver Erfahrungen“" verwendet, „die aus der Interaktion zweier Faktoren resultieren: der realen Ereignisse der äußeren Welt und der unbewussten Phantasien der inneren Welt" (Michels u. Roughton 1985, S. 645). Dieser Sachverhalt sei von Arlow (1969, S. 48) in der inzwischen klassisch gewordenen Metapher zweier Projektoren dargestellt worden, die von gegenüberliegenden Positionen aus auf die zwei Seiten einer durchsichtigen Leinwand zwei verschiedene Filme projizieren, die dort zu einem einheitlichen Film verschmelzen würden.

Angesichts des seit Jahren immer wieder kritisierten (zuletzt Goldberg 2002) Ausfransens psychoanalytischer Konzepte in eine Vielfalt heterogener Meinungen scheint dieser Konsens höchst erfreulich. Bei näherem Hinsehen wird allerdings frag- lich, ob der von Michels (1985) diagnostizierte Konsens wirklich besteht. Gewiss, es finden sich Autoren, die offensichtlich mit Arlow (1985, S. 521) Freuds Begriff für anachronistisch halten und psychische Realität so verstehen, wie sie von Arlow (1969, S. 43) definiert wurde, nämlich als „die ,wirkliche Erinnerung eines seelischen Ereignisses, welches immer eine Mixtur aus Fakten und Phantasien darstellt“.

Aber andere Psychoanalytiker verstehen unter diesem Begriff etwas ganz anderes. Beispielsweise ist Edelson (1988, S. 15) der Ansicht, dass sich Freuds Begriff auf „bewusste seelische Konstruktionen [,constructions']" bezieht, die auf der Grundlage bestimmter „Transformationsregeln“ und „nach besonderen, wunscherfüllenden Prinzipien" aus Erfahrungen gebildet werden. Beres (1968, S. 510) hingegen setzt die unbewussten seelischen Repräsentanzen mit dem Freud-Begriff gleich und sieht in den „seelischen Konstruktionen“ lediglich ihre Abkömmlinge. McLaughlin (1981, S. 642) ist der Ansicht, dass sich dieser Begriff bei Freud auf die „frühe, nach dem Primärvorgang operierende seelische Organisation eines Patienten" bezieht. Und nach Sandler u. Nagera (1963, S. 201) beschreibt „psychische Realität" bei Freud eine Eigenschaft unbewusster Phantasien, nämlich dass sie „behandelt werden, als wären sie reale Ereignisse“.

Mir scheint, dass diese Auffassungen von einem Konsens weit entfernt sind. Obgleich Fonagy (1995, S. 39) auf dem 1995 unter dem Titel „Psychic reality“ durch- 
geführten Kongress die gleiche Übereinstimmung der Auffassungen, wie Michels (1985), diagnostiziert: „In traditioneller Weise wird der Ausdruck, psychische Realität' von den meisten Psychoanalytikern benutzt, um die durch unbewusste Prozesse beeinflussten subjektiven Erfahrungen zu bezeichnen", kann auch zehn Jahre nach Michels (1985) Feststellung von einem Konsens nicht die Rede sein. Zwar verwenden, wie schon früher, auch nach 1985 einige Psychoanalytiker diesen Begriff im Sinne von Arlow (1996) und Fonagy (1995). Puget (1995, S. 29; Kursivierung, S. Z.) allerdings meint, dass sich „psychische Realität auf eine individuelle, phantastische, eingebildete, gewünschte, subjektive, unwirkliche, halluzinierte, unerkennbare Realität bezieht, die im Innern des psychischen Apparates von jeder Psyche konstruiert wird“. Nach Britton (1995, S. 20) wird „psychische Realität“ durch einen „bewussten oder unbewussten... Glauben“ hergestellt, „der den Status der psychischen Realität auf existierende mentale Gebilde (Phantasien) überträgt, und dadurch Glauben herstellt“. Nach Moore u. Fine (1990, S. 162) bezeichnet psychische Realität „die ganze subjektiv erfahrene Welt des Individuums, welche Gedanken, Gefühle und Phantasien ebenso einschließt wie die Wahrnehmungen der Außenwelt". Für Faimberg $(1995$, S. 9) bezieht sich dieser Begriff darauf, dass „unbewusste Wünsche (und unbewusste Phantasien) reale Effekte produzieren“. Torras de Beà (in: Penot 1996, S. 49; Kursivierungen aufgehoben, S.Z.) definiert, wie Edelson (1988) „psychische Realität als die inneren Repräsentanzen der Außenwelt und der Objekte“. Berenstein (1995, S. 3; s. auch Etchegoyen 1996) nimmt zu der Auffassung Edelsons (1988) die von Beres (1968) hin$\mathrm{zu}$, wendet die objektive Unterscheidung von Innen und Außen ins Subjektive und betrachtet psychische Realität als eine „Ansammlung von Erfahrungen, Emotionen und unbewussten Repräsentanzen, welche das Ich als zugleich Innen und als real fühlt". Und nach Frosch (2002, S. 604) bezieht sich psychische Realität nicht nur auf unbewusste Wünsche, sondern schließt „alle Manifestationen der Phantasie" ein.

Anstelle eines Konsenses findet man, wie beispielsweise auch Puget (1995, S. 29) feststellte, ein „Maximum an Mehrdeutigkeit", die auf diesem Kongress nicht ausgeräumt werden konnte, und die in einer "babylonischen Sprachverwirrung" (1995, S. 29) endete. Zu Recht hält Arlow (1996, S. 663) fest: „Während es scheint, dass das Konzept der psychischen Realität allgemein akzeptiert wird, gibt es keine Übereinstimmung darüber, woraus die psychische Realität zusammengesetzt ist".

Der Begriff wurde und wird für sehr unterschiedliche Inhalte verwendet. Trotz dieser Unterschiede sind einige der Autoren der Ansicht, dass sie diesen Begriff so verwenden, wie Freud ihn verstanden habe. Obwohl das Interesse an Freud im Verschwinden begriffen ist und die Aufforderung, $\mathrm{zu}$ seinen Konzeptualisierungen $\mathrm{zu}-$ rückzukehren, diskreditiert wird - z.B. von Schafer (1999) -, scheint es gleichwohl sinnvoll zu klären, was Freud mit „psychischer Realität" meinte, welches gegenwärtige Verständnis mit dem Freuds konsistent ist, und wie ein Konsens aussehen könnte.

\section{Freuds Begriff der psychischen Realität}

Nachdem Freud (1950c, S. 371) schon im „Entwurf“ „Denkrealität" und „externe“ Realität unterschied, erscheint der Begriff "psychische Realität" in veröffentlichter Form erstmals in der "Traumdeutung" und zwar als Gegensatz zur materiellen Realität. „Hat man die unbewussten Wünsche", schreibt Freud (1900a, S. 625), „auf ihren letzten und wahrsten Ausdruck gebracht, vor sich, so muss man wohl sagen, dass die psychische Realität eine besondere Existenzform ist, welche mit der materiellen Realität nicht verwechselt werden soll'“.

Im Hinblick auf die Erkennbarkeit wird das Unbewusste mit der realen Außenwelt gleichgesetzt. Einige Passagen vorher erläutert Freud (1900a, S. 617f.) das „Unbe- 
wusste“ als „das eigentliche reale Psychische, uns nach seiner inneren Natur so unbekannt wie das Reale der Außenwelt, und uns durch die Daten des Bewusstseins ebenso unvollständig gegeben wie die $\mathrm{Au}$ ßenwelt durch die Angaben unserer Sinnesorgane“. Da die bewussten Effekte einen vom unbewussten Prozess ganz abweichenden Charakter zeigen können, ist es für die „innere Wahrnehmung unmöglich, den einen als den Ersatz des anderen [zu] erkennen“ (1900a, S. 617). Deshalb warnt Freud mit Kant, „die subjektive Bedingtheit unserer Wahrnehmung nicht zu übersehen, und unsere Wahrnehmung nicht für identisch mit dem unerkennbaren Wahrgenommenen $\mathrm{zu}$ halten... die Bewusstseinswahrnehmung nicht an die Stelle des unbewussten psychischen Vorganges $\mathrm{zu}$ setzen, welcher ihr Objekt ist. Wie das Physische, braucht auch das Psychische nicht in Wirklichkeit so zu sein, wie es uns erscheint" (1915e, S. 270).

Obwohl Freud mit Kant darin übereinstimmt, dass unsere Wahrnehmungen nicht dem Wahrgenommenen entsprechen müssen, folgt daraus nicht, dass Freud ein Agnostiker war. Zwar sieht er die Erkennbarkeit des Unbewussten und der materiellen Realität in gleicher Weise eingeschränkt. Aber diese Einschränkung bezieht sich allein auf die Wahrnehmung durch unsere Sinnesorgane, d.h. auf die sinnliche Erkenntnis. Diese geht auch unmittelbar aus folgender Passage hervor: „Die Vorgänge, mit denen sie [die Psychoanalyse] sich beschäftigt, sind an sich ebenso unerkennbar wie die anderer Wissenschaften, der chemischen oder physikalischen, aber es ist möglich, die Gesetze festzustellen, denen sie gehorchen, ihre gegenseitigen Beziehungen und Abhängigkeiten über weite Strecken lückenlos zu verfolgen, also das, was man als Verständnis des betreffenden Gebiets von Naturerscheinungen bezeichnet" (1940a, S. 80 f.).

In dieser Definition des Unbewussten als dem real Psychischen wird das Unbewusste nicht nur hinsichtlich seiner Erkennbarkeit, sondern auch ontologisch, d.h. in dem Sinn bestimmt, dass es ebenso unabhängig vom Betrachter in der Seele vorhanden ist, wie die materielle Außenwelt außerhalb der Seele existiert. Unter Hinweis auf und im Einvernehmen mit Lipps, der, wie Freud, die Ansicht vertrat, dass das Unbewusste seelischer Natur ist, wendet Freud (1900a, S. 616; Kursivierungen, S. Z.) gegen die damalige akademische Psychologie, die das Unbewusste als biologisch verstand, ein, dass „unbewusste psychische Vorgänge..., der zweckmäßige und wohlberechtigte Ausdruck für eine feststehende Tatsache“" sind.

Offen ist jedoch, was Freud hier mit unbewusst bzw. unbewussten psychischen Vorgängen genau meint. Ausgehend von diesen und einer Reihe weiterer Textstellen bei Freud, in denen er feststellt, dass das Psychische an sich unbewusst ist, vor allem aber aus der Passage: „Das Psychische an sich, was immer seine Natur sein mag, ist unbewusst, wahrscheinlich von ähnlicher Art wie alle anderen Vorgänge in der Natur, von denen wir Kenntnis haben“ (1940b, S. 144), folgert z. B. Solms (1997a, S. 688; Kursivierungen aufgehoben, S. Z.), dass Freud mit unbewusst „psychische Energie, Besetzung, Abfuhr, und Ähnliches" meine, mit denen er die "die in uns stattfindenden natürlichen Prozesse" beschreibe. Sie wären als Äquivalent der „Teilchen, Wellen, Energien, Kräfte und Ähnlichem" konzeptualisiert, mit denen Physiker die „um uns herum stattfindenden natürlichen Prozesse" beschrieben. Sie wären ebenfalls „,an sich unbewusst", und stellten, wie diese, einen „Versuch“ dar, „die unerkennbaren Prozesse zu beschreiben, die hinter der bewussten Wahrnehmung [,perceptual awareness'] liegen“" (1997a, S. 687f.).

Folgt man dieser Auffassung, müsste man annehmen, dass Freud (1900a, S. 617) entgegen seiner zentralen These, dass das Unbewusste „das eigentliche reale Psychische" ist, das Unbewusste letztlich doch in den natürlichen Prozessen der Biologie lokalisiert. Auf den ersten Blick scheint es zwar, als könnte man zur Unterstützung die- 


\section{Zusammenfassung $\cdot$ Abstract}

Forum Psychoanal 2006 · 22:240-248

DOI 10.1007/s00451-006-0287-4

C) Springer Medizin Verlag GmbH 2006

\section{Siegfried Zepf}

\section{Das Konzept der "psychischen Realität"}

\section{Zusammenfassung}

Untersucht wird, inwieweit das gegenwärtige Verständnis der „psychischen Realität" mit der Konzeptualisierung Freuds noch identisch ist und das Konzept einheitlich verwendet wird. Es wird aufgewiesen, dass dieser Begriff in der gegenwärtigen Literatur auf sehr verschiedene Inhalte - z. B. Ersatzbildungen, von unbewussten Prozessen beeinflusstes subjektives Erleben, erste psychische Organisation des Subjekts, Manifestationen von Phantasien - bezogen wird. Bei Freud

hingegen bezieht sich dieser Begriff in zweifacher Weise auf unbewusste Inhalte: Zum einen auf ihre reale Existenz und zum anderen auf die Eigenschaft unbewusster Vorstellungen, auf reale Ereignisse der Außenwelt auch dann zu verweisen, wenn sie ursprünglich bloße Phantasien waren. Es wird dafür plädiert, dieses Konzept nicht zu verwässern und die Definition Freuds beizubehalten.

\section{The concept of "psychic reality"}

\section{Abstract}

The questions are examined, to what extent our contemporary understanding of "psychic reality" and Freuds concept are still identical and whether this concept is used always in the same sense. It can be shown, that in the present literature this concept relates to very different contents as for example substitutive formations, subjective experiences being influenced by unconscious processes, the first mental organization of the

subject or, simply, manifestations of phantasies. In Freud's view, however, this concept refers twofold to unconscious contents: on the one hand to their real existence, on the other hand to the quality of unconscious concepts, to refer even then to real events in the external world, if, originally, they were only phantasies. It is argued not to blur the original concept and to retain the definition of Freud. 
ser These noch darauf verweisen, dass

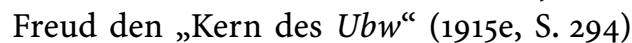
nach 1912 in der urverdrängten, nie bewusst gewordenen archaischen Erbschaft des Menschen (1912-13a, S. 212, S. 345) sieht, die sich in ihre DesoxyribonukleinsäureStrukturen eingetragen hat. Aber bei diesen „Erinnerungsspuren an das Erleben früherer Generationen“ (1939a, S. 206) handelt es sich Freud (1915e, S. 294; Kursivierung, S. Z.) zufolge nicht um einen biologischen Sachverhalt, sondern um „ererbte psychische Bildungen“.

Gleichgültig wie man zu Freuds These einer archaischen Erbschaft stehen mag, dass es sich hierbei um psychische Bildungen handelt, geht auch aus der Formulierung hervor, dass das in der Ontogenese Verdrängte seiner „Natur nach von dem Ererbten nicht verschieden $\mathrm{zu}$ sein braucht" (1915e, S. 294). Wie Brenner (1957; s. auch Maze u. Henry 1996) anmerkt, ist nach 1926 auch nicht mehr von einer „Urverdrängung“, sondern nur mehr von „ersten und ursprünglichen“ Verdrängungen die Rede, die alle in der frühen Kindheit geschehen (z. B. Freud 1933a, S. 101; 1937c, S. 71). Auch aus Brenners (1957, S. 42) Schlussfolgerung, dass sich im Verständnis Freuds die Gründe und Mechanismen der kindlichen oder Urverdrängung nicht wesentlich von denen der späteren Verdrängungen unterscheiden, geht hervor, dass in Freuds Auffassung das Verdrängte nicht biologischen, sondern seelischen Charakter aufweist.

Während ferner in der Auffassung Freuds psychische Vorgänge den Vorgängen in der Natur nur im Hinblick auf ihre sinnliche Wahrnehmung ähnlich, sonst aber qualitativ voneinander verschieden sind, werden sie von Solms (1997a) nicht nur in dieser Hinsicht, sondern auch in ihrer Qualität als identisch aufgefasst. Des Weiteren werden von ihm, wie auch Cavell (1997, S. 721) feststellt, „unbewusst“ und „sinnlich nicht erkennbar" fälschlicherweise gleichgesetzt. Die „natürlichen Elemente", die der Physiker mit den Begriffen „Teilchen, Wellen, Energien, Kräften und Ähnlichem“ beschreibt, sind jedenfalls nicht, an sich un- bewusst“. Vielmehr sind sie der sinnlichen Erkenntnis nicht unmittelbar zugänglich. Die Gesetze, denen die Prozesse in der äußeren Natur unterliegen, sind ebenso Abstraktionen, Resultat des abstrakt-theoretischen Denkens und sinnlich ebenso wenig unmittelbar erkennbar, wie die Prozesse Abstraktionen sind, denen Freud zufolge die psychischen Vorgänge unterliegen - z. B. freie und gebundene Energie, Besetzung, Überbesetzung - und die sich der unmittelbaren sinnlichen Erkenntnis des Patienten und auch des Psychoanalytikers entziehen. Genau auf diese Abstraktionen, die das sinnlich Wahrnehmbare erklären sollen, verweist Freud (1940a, S. 85 f.) in einer Passage, in der er auf die Frage nach dem Unterschied von Unbewusstem und Vorbewussten antwortet: „Wir nehmen an, wie wir von anderen Naturwissenschaften gewohnt sind, dass im Seelenleben eine Art von Energie tätig ist, aber es fehlen uns alle Anhaltspunkte, uns ihrer Kenntnis durch Analogien mit anderen Energieformen zu nähern. Wir glauben, zu erkennen, dass die nervöse oder psychische Energie in zwei Formen vorhanden ist, einer leicht beweglichen und einer eher gebundenen, sprechen von Besetzungen und Überbesetzungen der Inhalte und wagen selbst die Vermutung, dass eine ,Überbesetzung' eine Art von Synthese verschiedener Vorgänge herstellt, bei der die freie Energie in gebundene umgesetzt wird. Weiter sind wir nicht gekommen, immerhin halten wir an der Meinung fest, dass auch der Unterschied des unbewussten von einem vorbewussten Zustand in solchen dynamischen Verhältnissen liegt...".

Auch heißt es in derselben Arbeit, in der vom „Kern des $U b w$ “ die Rede ist, dass im Unbewussten die „Sachvorstellung[en]“ enthalten sind, die die ihnen „zugehörigen Wortvorstellung[en]" verloren haben (1915e, S. 300). Und diese Triebrepräsentanzen sind nicht von vornherein unbewusst. Vielmehr wurden sie im Zuge des Lebens unbewusst und können in der Auffassung Freuds (1915e, S. 281 f.) in der sie verbergenden Form von „Ersatzvorstellungen" wieder im Bewusstsein erscheinen. 
Aus meiner Sicht verweist Freuds ontologische Definition des Unbewussten nicht - wie Solms (1997a, S. 688) glaubt - auf „psychische Energie, Besetzung, Abfuhr und Ähnliches“, sondern auf diese unbewusst gewordenen Sachvorstellungen, d.h. auf die Tatsache, dass Sachvorstellungen, die die ihnen zugehörigen Wortvorstellungen verloren haben, ebenso unabhängig vom Betrachter in der Seele vorhanden sind, wie die materielle Außenwelt außerhalb der Seele existiert.

Die zweite Bestimmung des Unbewussten als psychische Realität ist eine qualitative. Sie bestimmt dessen Inhalte als Inhalte, auf die das Subjekt reagiert, als wären in ihnen Ereignisse abgebildet, die real stattgefunden haben. Weil im Unbewussten eine „Ersetzung der äußeren Realität durch die psychische“" (1915e, S. 286) erfolgt, erhalten dessen Inhalte selbst einen „Realitätswert“ (1927c, S. 354; Laplanche u. Pontalis 1967, S. 426). „Der befremdendste Charakter der unbewussten (verdrängten) Vorgänge“, schreibt Freud (1911b, S. 237), „ergibt sich daraus, dass bei ihnen die Realitätsprüfung nichts gilt, die Denkrealität gleichgesetzt wird der äußeren Wirklichkeit, der Wunsch der Erfüllung, dem Ereignis“.

Diese „Denkrealität“ ist für die Neurose entscheidend: „Die Realität der Neurose ist nämlich die Denkrealität und nicht die äußerliche Realität“ (Freud am 16.03. 1910, in: Nunberg u. Federn 1967, S. 413) und: „Die Neurose ist dadurch charakterisiert, dass sich die psychische Realität über die faktische setzt, auf Gedanken ebenso ernsthaft reagiert wird wie die Normalen nur auf Wirklichkeiten" (1912-13a, S. $191 \mathrm{f}$.).

Zum einen erweist sich also bei Freud „psychische Realität“ als eine Tatsachenfeststellung in dem Sinn, dass es ein seelisch Unbewusstes wirklich gibt. Zum anderen bezieht sich dieser Begriff nicht auf die biologische Realität der Triebe; er ist kein Synonym für eine phantastische oder halluzinierte, prinzipiell unerkennbare Realität, für die vom Subjekt erfahrene Welt, die sowohl Gedanken, Gefühle und
Phantasien als auch Wahrnehmungen der Umwelt einschließt, für die seelische Organisation des Subjekts am Beginn seines Lebens, für alle Manifestation der Phantasie, für seelische Repräsentanzen, die nach dem Prinzip der Wunscherfüllung konstruiert wurden, für den Ausdruck subjektive Realität oder für Erfahrungen, Gefühle und unbewusste Repräsentanzen, die ein Subjekt als real und ihm zugehörig fühlt, und auch die Gleichsetzung von psychischer Realität und unbewussten seelischen Repräsentanzen greift zu kurz. Zwar betont diese Gleichsetzung die reale Existenz unbewusster Inhalte. Es sind jedoch nicht unbewusste Wünsche (und unbewusste Phantasien), die reale Effekte hervorrufen. Entscheidend ist, wie Sandler u. Nagera (1963) zu Recht schreiben, dass seelische Inhalte, die unbewusst werden, für das Subjekt auf reale Vorkommnisse verweisen, obwohl es sich in Wirklichkeit um Phantasien handeln kann. Es ist mithin nicht nur der unbewusste Charakter der Phantasien, die in der Neurosenbildung an zentraler Stelle steht; es ist zugleich die "phantasierte Realität“ (Arlow 1985, S. 534) ihrer Inhalte. Auch die unbewusst gewordenen Phantasien, sagt Freud (1916-17a, S. 383), „besitzen eine Art von Realität... und diese Tatsache hat kaum geringere Bedeutung für seine Neurose, als wenn er den Inhalt dieser Phantasien wirklich erlebt hätte. Diese Phantasien besitzen psychische Realität im Gegensatz zur materiellen".

Diese Phantasien gewinnen einen psychischen Realitätsstatus allein dadurch, dass sie unbewusst wurden, und nicht, wie Britton (1995, S. 19) meint, in einem unbewusst ablaufenden Glaubensakt, der ihnen erst die „Kraft der Realität“ zuschreibt.

Diese Auffassung ist auch aus folgendem Grund problematisch. Unter Bezug auf Flew (1979, S. 38) definiert Britton (1995, S. 21) Glauben als die „epistemologische Haltung, eine Annahme $\mathrm{p}$ auch dann für wahr zu halten, wenn es dafür zwar einen gewissen Grad von, aber noch keine sichere Evidenz gibt. Während aus dem Wissen um $\mathrm{p}$ folgt, dass $\mathrm{p}$ wahr ist, folgt 
aus dem Glauben an $\mathrm{p}$, dass $\mathrm{p}$ in Wirklichkeit falsch ist". Wie aber soll eine solche Operation im Unbewussten möglich sein, in dem der „Primärvorgang“ herrscht (Freud 1915e, S. 286), auf dessen Denkniveau es „Realitätszeichen nicht gibt, so dass man die Wahrheit und die mit Affekt besetzte Fiktion nicht unterscheiden kann" (Brief vom 21.09. 1897, in: Masson 1985, S. 284)? Hier werden "Gegensätze nicht auseinandergehalten“, sondern wie identisch behandelt, die „entscheidenden Regeln der Logik haben keine Geltung" (Freud 1940a, S. 91), es gibt „keine Negation, keinen Zweifel, keine Grade von Sicherheit" (Freud 1915e, S. 285). Freud selbst jedenfalls war der Ansicht, dass „Glauben (Zweifel) ein Phänomen [ist], das ganz dem System des Ich $(B w)$ angehört und kein Gegenstück im Ubw hat" (Manuskript N, 31.05. 1897, in: Masson 1985, S. 268).

\section{Psychische Realität und Ersatzbildungen}

Nicht unter den Begriff der psychischen Realität werden von Freud (1900 a, S. 625) die „Übergangs- und Zwischengedanken“ subsumiert. Ihnen, sagt Freud (1900a, S. 625), ist die Realität „natürlich abzusprechen“. Sie gehören der „Innenwelt“ $\mathrm{zu}$ und vertreten sowohl die innere Welt als auch die „Außenwelt als ihr Abbild“ (1924 b, S. 389). Sie sind zwar in ontologischem Sinn real - auf diese Realität beziehen sich Edelsons (1988), Torras de Beás (in: Penot 1996) und Pugets (1995) Formulierungen -, aber in qualitativer Hinsicht sind sie das „Nichtreale, bloß Vorgestellte, Subjektive“; sie sind „nur innen“, während „das andere, Reale, auch im Draußen vorhanden" ist (Freud 1925h, S. 13). Ob zwischen den „Übergangs- und Zwischengedanken" und der Außenwelt eine Abbildrelation besteht, lässt sich vom Subjekt mithilfe seiner „Realitätsprüfung“ (1925h, S. 13) entscheiden - mit ihr wird festgestellt, „ob etwas im Ich als Vorstellung Vorhandenes auch in der Wahrnehmung (Realität) wieder gefunden werden kann“ (1925h, S. 13) -, sodass diese Prüfung Auskunft gibt, ob es sich bei diesen Übergangs- und Zwischengedanken" um Phantasien oder um Vorstellungen von realen Ereignissen handelt, die in der Gegenwart stattfinden bzw. in der eigenen Vergangenheit stattfanden.

Durch ihre Verbindung mit der unbewusst gewordenen Vergangenheit ergibt sich ihre ontologische Realität als Symbol im Sinn von Jones (1916). ${ }^{1}$ Wie Major (1980, S. 461) schreibt, zeichnet sie der Realitätstyp aus, den „Freud den Gedanken zuschrieb, welche für die Besetzung genutzt wurden, d.h. Zwischengedanken. Diese Gedanken gehören weder der psychischen noch der äußeren Realität an; sie repräsentieren vielmehr eine symbolische Verbindung dieser beiden Realitäten“. Anders ausgedrückt und wie auch Anzieu (1986, S. 225) betont: „Im Hinblick auf die unbewusst gewordene Vergangenheit haben die Übergangs- und Zwischengedanken“ den Charakter von „Ersatzvorstellungen“, auf die in Freuds Auffassung die den unbewusst gewordenen Sachvorstellungen teilweise entzogene Besetzung verschoben und als Gegenbesetzung verwendet wird (1915e, S. 28o f.). In ihnen werden die unbewussten, für real gehaltenen Ereignisse im Gewand anderer realer Ereignisse bewusstseinskonform mystifiziert zur Darstellung gebracht.

Hier zeigt sich, dass in Arlows (1969) Analogie zweier Projektoren, die von gegenüberliegenden Positionen aus auf die zwei Seiten einer durchsichtigen Leinwand zwei verschiedene Filme projizieren, die dort $\mathrm{zu}$ einem einheitlichen Film verschmelzen, nicht die psychische Realität, sondern die Ersatzbildungen metaphorisch dargestellt werden. Ersatzvorstellungen sind das Scharnier, in dem sich die innere, psychische und äußere materielle Realität in Bildung ihres subjektiven Erlebens verbinden. Auch wenn Michels (1985)

\footnotetext{
1 Jones' (1916, S. 116) bekannte Statements lauten: "Nur das Verdrängte wird symbolisiert" und "Nur das Verdrängte muss symbolisiert werden".
} 
fälschlicherweise Freuds Begriff der psychischen Realität im Biologischen verankert, so ist die folgende Formulierung doch mit dem Verständnis Freuds konsistent: „Die psychische Realität ist weniger die subjektive Erfahrung selbst, sondern vielmehr die innere Quelle subjektiver Erfahrung - so wie die äußere Realität die äußere Quelle subjektiver Erfahrung ist" (Michels u. Roughton 1985, S. 645).

\section{Zusammenfassende Schlussbemerkungen}

„Psychische Realität" meint bei Freud Vorstellungen, die unbewusst wurden, und die dadurch in dem Sinn selbst einen „Realitätswert" bekommen, dass sie auf reale Ereignisse verweisen, und zwar gleichgültig, ob sich in ihnen reale Ereignisse ab- oder nicht abbilden. Aus mehreren Gründen sollte an diesem Konzept festgehalten werden. Zunächst macht die ontologische Bestimmung des Unbewussten als eine von seiner Wahrnehmung unabhängigen Realität deutlich, dass das Gegenstandsverständnis Freuds mit dem in den letzten Jahren in der Psychoanalyse zunehmend in Mode kommenden Konstruktivismus ${ }^{2}$ und der damit verbundenen konstruktivistischen Fassung des Unbewussten nicht kompatibel ist. Ferner folgt aus dem generellen „Realitätswert" unbewusst gewordener Vorstellungen, dass es kein Kriterium geben kann, anhand dessen wahre von falschen Erinnerungen unterschieden werden können. Im Zuge von Ersatzbildungen können frühere reale Vorkommnisse für Phantasien und frühere Phantasien für reale Ereignisse gehalten werden.

Entscheidender aber für das Beibehalten des Konzeptes der psychischen Realität ist, dass sich dieses Konzept in systematischer Weise mit dem Konzept der Ersatz-

2 In Freuds (1915e, S. 270) Sicht erscheint die epistemologische Position des Konstruktivismus „als eine weitere Fortbildung des primitiven Animismus, der uns überall Ebenbilder unseres Bewusstseins vorspiegelte....". bildungen verbindet, eine Verbindung, in der im Verständnis Freuds die Einheit und die Verschiedenheit der Neurosen gründen. ${ }^{3}$ Das gemeinsame Moment der Neurosen liegt darin, dass Vorstellungen unbewusst werden und sich in Gestalt von Ersatzbildungen wieder im Bewusstsein präsentieren, deren Inhalte dann in mystifizierter Form auf etwas verweisen, das subjektiv in der Vergangenheit zu Recht oder zu Unrecht einen „Realitätswert“ besaß bzw. besitzt, und die Verschiedenheit der Neurosen gründet in den besonderen Abwehrmechanismen, mit denen die Ersatzbildungen hergestellt werden.

Weil den Ersatzbildungen nicht anzusehen ist, in welcher Form sie etwas ersetzen, macht das Konzept der psychischen Realität des Weiteren deutlich, dass die inhaltlichen Deutungen der eigenen Lebensgeschichte ebenso verfälscht sind wie die emotiv-affektiven Deutungen der äußeren Realität. Wie etwa das Beispiel der Pferdephobie des kleinen Hans (Freud 1909b) zeigen kann, werden die Objekte der äußeren Realität nicht verkannt. Wohl können jene ihrer Verhaltensaspekte eine emotivaffektive Bedeutung für das Subjekt gewinnen, in denen sich das unbewusst gewordene Kindheitsdrama in entstellter Form darstellt. Diese Verfälschungen, die durch unbewusste Inhalte zustande kommen, zu korrigieren, ist aber fraglos das Ziel einer psychoanalytischen Behandlung. Weil das Konzept der psychischen Realität, wie es von Freud gefasst wurde, mithin in der Tat das Gebiet der Psychoanalyse definiert, bin ich im Gegensatz zu Arlow (1985) der Ansicht, dass das Konzept Freuds nicht aufgegeben, sondern in der von ihm definierten Form beibehalten werden sollte.

\footnotetext{
${ }^{3}$ Indem Freud "Verdrängung" als Oberbegriff für "Abwehrmechanismen" gebraucht, sagt er anlässlich eines Vortrags von Adler "Über die Einheit der Neurosen“: „Er fasse die Neurosen als Ersatzbildungen für die verdrängte Libido auf und erkläre inre Verschiedenheit durch die verschiedenen Mechanismen der Verdrängung und der Wiederkehr des Verdrängten" (Freud am 02. 06. 1909, in: Nunberg u. Federn 1967, S. 242).
} 


\section{Anschrift}

\section{Univ.-Prof. em. Dr. med. Siegfried Zepf}

Narzissenstrasse 5, 66119 Saarbrücken

E-Mail: s.zepf@rz.uni-sb.de

\section{Literatur}

Anzieu D (1986) The place of Germanic language and culture in Freud's discovery of psychoanalysis between 1895 and 1900 . Int J Psychoanal 67:219-226

Arlow JA (1969) Fantasy, memory, and reality testing. Psychoanal Q 38: 28-51

Arlow JA (1985) The concept of psychic reality and related problems. J Am Psychoanal Assoc 33:521-535

Arlow JA (1996) The concept of psychic reality - How useful? Int J Psychoanal 77:659-666

Berenstein I (1995) Psychic reality and clinical technique. Int J Psychoanal 76:3-7

Beres D (1968) The humanness of human beings: Psychoanalytic considerations. Psychoanal Q 7:487-522

Brenner C (1957) The nature and development of the concept of repression in Freud's writings. Psychoanal Stud Child 12: $19-46$

Brenner C (2000) Observations on some aspects of current psychoanalytic theories. Psychoanal Q 69:597-632

Britton R (1995) Psychic reality and unconscious belief. Int J Psychoanal 76:9-23

Cavell M (1997) Commentaries. J Am Psychoanal Assoc 45: 721-726

Edelson M (1988) Psychoanalysis: a theory in crisis. University Chicago Press, Chicago

Etechegoyen RH (1996) Some views on psychic reality. Int J Psychoanal 77:1-14

Faimberg H (1995) Misunderstandings and psychic truth. Int J Psychoanal 76:9-13

Flew A (ed) (1979) A dictionary of philosophy. Macmillan, London

Fonagy P (1995) Playing with reality: the development of psychic reality and its malfunction in borderline personalities. Int J Psychoanal 76:39-44

Freud S (1900a) Die Traumdeutung. Gesammelte Werke, Bde 2/3, Fischer, Frankfurt aM

Freud S (1909b) Analyse der Phobie eines fünfjährigen Knaben. GW Bd 7, S 241-377

Freud $S(1911 b)$ Formulierungen über zwei Prinzipien des psychischen Geschehens. GW Bd 8, S 229-238

Freud S (1912-1913a) Totem und Tabu. GW Bd 9

Freud S (1915e) Das Unbewusste. GW Bd 10, S 263-303

Freud S (1916-1917a) Vorlesungen zur Einführung in die Psychoanalyse. GW Bd 11

Freud S (1924b) Neurose und Psychose. GW Bd 13, S 385-391

Freud S (1925h) Die Verneinung. GW Bd 14, S 9-15
Freud S (1927c) Die Zukunft einer Illusion. GW Bd 14, S $323-380$

Freud S (1933 a) Neue Folgen der Vorlesungen zur Einführung in die Psychoanalyse. GW Bd 15

Freud S (1937 c) Die endliche und die unendliche Analyse. GW Bd 16, S 57-99

Freud S (1939a) Der Mann Moses und die monotheistische Religion. GW Bd 16, S 101-246

Freud S (1940a) Abriss der Psychoanalyse. GW Bd 17, S 63-138

Freud S (1940 b) Some elementary lessons in psycho-analysis. GW Bd 17, S 139-147

Freud S (1950c) Entwurf einer Psychologie. In: Bonaparte M, Freud A, Kris E (Hrsg) (1950) Aus den Anfängen der Psychoanalyse 1887-1902. Fischer, Frankfurt aM, S 297-384 (1975)

Frosch A (2002) Transference. Psychoanal Psychol 19:603-633

Goldberg A (2002) American pragmatism and American psychoanalysis. Psychoanal Q 71:235-250

Jones E (1916) The theory of symbolism. In: Jones E (1961) Papers on psychoanalysis. Beacon Press, Boston, pp 87-144

Laplanche J, Pontalis JB (1967) Das Vokabular der Psychoanalyse. Suhrkamp, Frankfurt aM (1972)

Major R (1980) The voice behind the mirror. Int Rev Psychoanal 7:459-467

Masson JM (Hrsg) (1985) Sigmund Freud. Briefe an Fließ 1887-1904. Ungekürzte Ausgabe. Fischer, Frankfurt aM (1986)

Maze JR, Henry RM (1996) Problems in the concept of repression and proposals for their resolution. Int J Psychoanal 77: 1085-1100

McLaughlin JT (1981) Transference, psychic reality and countertransference. Psychoanal Q 50:639-664

Michels R (1985) Introduction to panel: perspectives on the nature of psychic reality. J Am Psychoanal Assoc 33:515-519

Michels R, Roughton R (1985) Perspectives on the nature of psychic reality. J Am Psychoanal Assoc 33:645-659

Moore B, Fine B (1990) Psychoanalytic terms and concepts. Yale University Press, New Haven

Nunberg H, Federn E (Hrsg) (1967) Protokolle der Wiener Psychoanalytischen Vereinigung, Bd II: 1908-1910. Fischer, Frankfurt aM (1977)

Penot B (1996) Panel report: the patient's psychic reality. Int J Psychoanal 77:49-52

Puget J (1995) Psychic reality or various realities. Int J Psychoanal 76:29-34

Sandler J, Nagera H (1963) Einige Aspekte der Metapsychologie der Phantasie. Psyche - Z Psychoanal 20:188-221 (1966)

Schafer R (1999) Interpreting sex. Psychoanal Psychol 16:502-513

Solms M (1997a) What is consciousness? J Am Psychoanal Assoc 45:681-703

Solms M (1997b) Commentaries: response by Mark Solms. J Am Psychoanal Assoc 45:765-778

Torras de Beà E (1996) In: Pent B (reporter) Panel report: The patient's psychic reality. Int J Psychoanal 77:49-52 